\title{
Neutrino experiments at nuclear reactors
}

\author{
Jim Napolitano*† \\ Temple University \\ E-mail: tuf43817@temple.edu
}

\begin{abstract}
We review neutrino experiments using nuclear reactors as $\bar{v}_{e}$ sources, including the recent history, some current results, and upcoming experiments. After discussing production and detection of reactor neutrinos, various physics topics are covered including neutrino oscillations to measure $\theta_{12}$ and $\theta_{13}$, details of the reactor neutrino spectrum, sterile neutrinos and very short baseline experiments, and an upcoming experiment to measure the neutrino mass hierarchy. The author is a member of the Daya Bay Reactor Neutrino Experiment, and also the Precision Oscillation and Spectrum (PROSPECT) experiment, but coverage is provided of the recent and future suite of reactor experiments.
\end{abstract}

Flavor Physics and CP Violation,

6-9 June 2016

Caltech, Pasadena CA, USA

\footnotetext{
* Speaker.

${ }^{\dagger}$ Representing the Daya Bay and PROSPECT collaborations.
} 


\section{Production and Detection of Reactor $\bar{v}_{e}$}

Nuclear reactors are intense sources of electron antineutrinos $\bar{v}_{e}$. (Where there is no danger of confusion, we will simply use the term "neutrinos.") They are a product of the (neutron-rich) radioactive fission fragment isotopes, and the flux can be estimated from a knowledge of the reactor thermal power, the energy released per fission, and the number $(\sim 6)$ of fragment beta decays. Indeed, this was the source used to in fact discover the neutrino [1, 2, 3, 4]

Determining the flux precisely, however, and predicting the shape of the $\bar{v}_{e}$ energy spectrum with any accuracy, is actually quite difficult [5]. The distribution of fission fragments is not very well known, and can depend significantly on the incident neutron energy. Furthermore, the beta decay spectra, including branching ratios, endpoint energies, and degree of forbidden-ness, remain poorly known for many important nuclei. In addition, for nuclear power plant reactors, there is energy released from ${ }^{235} \mathrm{U},{ }^{239} \mathrm{Pu},{ }^{241} \mathrm{Pu}$, and (with fast neutrons) ${ }^{238} \mathrm{U}$, where the relative size of their contributions is not well known and changes with time.

Nevertheless, it is possible to get around these difficulties, and carry through important and precise measurements, and these methods and results are discussed in this talk. Furthermore, studies of the reactor spectra alone can be enlightening, and these topics are also included here.

All experiments use, essentially, the same method as Reines and Cowan to detect $\bar{v}_{e}$, namely the "inverse beta decay" (IBD) reaction on protons:

$$
\bar{v}_{e}+p \rightarrow e^{+}+n
$$

The cross section for this process is very well known [6]. An experiment typically uses a large volume of liquid scintillator, which is based on long hydrocarbon chains with a roughly 2:1 $\mathrm{H}: \mathrm{C}$ ratio, providing a proton target. The positron deposits energy in the scintillator, which converts it to light that is detected in photomultiplier tubes. The positron (quickly) annihilates against an atomic electron, contributing an additional $1.022 \mathrm{MeV}$ to its kinetic energy. This total signal is called the prompt energy, and (ignoring the neutron recoil energy) equals the incident neutrino energy, minus $0.78 \mathrm{MeV}$, accounting for the $n-p$ mass difference. The normally detectable neutrino energy spectrum stretches from about $1 \mathrm{MeV}$ up to $\approx 8 \mathrm{MeV}$, with a mean energy near $4 \mathrm{MeV}$.

The difficulty, of course, is background from ambient radioactivity and cosmic radiation. A many-ton detector hundreds of meters from the core of a power plant reactor, will only observe hundreds of IBD events per day. The background rate, on the other hand, is many orders of magnitude higher. There can be some abatement by putting the detectors within some kind of passive volume to shield against radioactivity, and overburden and some active detectors can reduce cosmic ray signals. These measures are not enough, though, so an additional handle is needed.

That handle is the delayed energy signal from neutron capture. The final state neutron in (1.1) thermalizes in the hydrogenous liquid scintillator volume, and will capture on either a proton or some doping isotope. Capture on a proton gives a $2.2 \mathrm{MeV}$ gamma ray, while doping the scintillator with gadolinium (which has a very large neutron capture cross section) gives an $\sim$ $8 \mathrm{MeV}$ signal. The capture time varies on the doping material and concentration, but is typically tens of microseconds. Other doping materials include ${ }^{6} \mathrm{Li}$, which gives a highly localized signal from $n+{ }^{6} \mathrm{Li} \rightarrow \alpha t$, and cadmium, which was used by Reines and Cowan. A different technique, capturing the neutrons on ${ }^{3} \mathrm{He}$-filled proportional counters, has also been employed [7]. 


\section{Primary Physics Goals and the Experimental Landscape}

After the discovery of the neutrino, the main intellectual interest in reactor neutrino physics has been to search for neutrino oscillations. Reactor neutrinos are too low in energy to make any lepton species other than the electron, so one can only search for $\bar{v}_{e}$ disappearance at reactors, where the $\bar{v}_{e}$ oscillates into some other species, reducing the observed $\bar{v}_{e}$ flux in an energy and baseline dependent way. The full expression for $\bar{v}_{e}$ disappearance, allowing for three generational mixing, is [8]

$$
\begin{aligned}
P_{e e}=1 & -\left\{\operatorname { c o s } ^ { 4 } ( \theta _ { 1 3 } ) \operatorname { s i n } ^ { 2 } ( 2 \theta _ { 1 2 } ) \operatorname { s i n } ^ { 2 } \left(\left(\Delta_{21}\right)\right.\right. \\
& +\cos ^{2}\left(\theta_{12}\right) \sin ^{2}\left(2 \theta_{13}\right) \sin ^{2}\left(\Delta_{31}\right) \\
& \left.+\sin ^{2}\left(\theta_{12}\right) \sin ^{2}\left(2 \theta_{13}\right) \sin ^{2}\left(\Delta_{32}\right)\right\}
\end{aligned}
$$

where

$$
\Delta_{i j}=1.27\left|\delta m_{j i}^{2}\right| \frac{L}{E_{v}} \quad \text { and } \quad \delta m_{j i}^{2}=m_{j}^{2}-m_{i}^{2}
$$

and neutrino masses are expressed in $\mathrm{eV}$ and $L / E_{V}$ in meters $/ \mathrm{MeV}$.

If the neutrino masses were known, or at least we knew the mass-squared-differences $\delta m_{j i}^{2}$, we could measure one of the mixing angles by placing a detector at a distance corresponding to $\Delta_{i j}=$ $\pi / 2$ for the nominal neutrino energy. This maximizes the disappearance signal. For the longest time, however, we had no guidance on what were the neutrino masses, so reactor disappearance experiments searched the parameter space, with, essentially, no luck. Until the end of the 20th century, reactor neutrino oscillation experiments all gave null results.

Of course, one limiting factor for these experiments was an accurate knowledge of the reactor flux and spectrum shape, particularly for small mixing angles. To the extent that the spectrum could be calculated, a measurement could be compared to prediction, but this was only a reliable approach if the spectrum could be determined with the appropriate precision.

The current generation of reactor neutrino oscillation experiments starts with Chooz [9]. This experiment, at a nuclear power plant in northern France, was located about $1 \mathrm{~km}$ from the reactor cores, under a significant rock overburden. No mass parameters were known at the time, so the location was far as practical given various constraints, but their measurement led to the most stringent limits at the time on $\theta_{13}$.

Positive results on reactor neutrino disappearance were first obtained by KamLAND [10], who extracted $\theta_{12}$, using a very long baseline. A trio of experiments aimed at measuring $\theta_{13}$, Daya Bay [11], RENO [12], and Double Chooz [13], have obtained positive results in the past few years. The next generation measurement will be by JUNO [14], obtaining precise values of $\theta_{12}, \delta m_{21}^{2}$, and $\delta m_{32}^{2}$, and determining the mass hierarchy through $\theta_{12}-\theta_{13}$ interference. These experiments will be reviewed in more detail below.

There has been an important interplay between reactor, accelerator, solar, and atmospheric neutrino oscillation experiments, and this has been largely responsible for the excellent progress in the past two decades. Two long standing issues, the Solar Neutrino Problem and the Atmospheric Neutrino Anomaly, were resolved in terms of neutrino oscillations by SNO and Super-K. (Consider, for example, the 2015 Nobel Prize in Physics.) KamLAND then demonstrated the so-called "large mixing angle" solution to the observations by SNO, determining both $\theta_{12}$ and $\delta m_{21}^{2}$, and accelerator 
experiments K2K and MINOS confirmed Super-K and provided precise values for both $\theta_{23}$ and $\delta m_{32}^{2}$. These results in turn determined $\delta m_{31}^{2}=\delta m_{32}^{2}-\delta m_{21}^{2}$ which allowed accurate placement of detectors to optimally search for $\theta_{13}$.

Other physics is also pursued with reactor neutrinos. The new generation of experiments obtains very high statistics samples that allow careful measurements of the reactor flux and energy spectrum. These are also discussed below, along with a new suite of very short baseline experiments at research reactors, currently under construction. Research reactors are attractive because their cores are very well understood, with fission yields dominated by one isotope, ${ }^{235} \mathrm{U}$. They also, unfortunately, have far less power than do nuclear generating station reactors.

Anomalies in the reactor neutrino flux, combined with other experiments, have inspired predictions of sterile neutrinos species. Indeed, the new generation of very short baseline reactor experiments are motivated by a desire to observe oscillations with wavelengths on the order of meters, disappearance oscillations from mixing with standard model neutrino species.

\section{KamLAND, Measuring $\theta_{12}$, and Searching for $\theta_{13}$}

With the demonstration from the Sudbury Neutrino Observatory (SNO) that solar neutrinos were in fact undergoing flavor transformation [15], the next goal was to determine the values of the neutrino oscillation parameters responsible for the observations. It was already known that electron densities in the Sun could dramatically enhance neutrino oscillations [16, 17], through the so called MSW Effect, but there was more than one solution in terms of neutrino oscillations.

The so-called "large mixing angle" solution, which implied a very large mixing angle $\theta_{12}$ and a very small mass difference $\delta m_{21}^{2} \sim 10^{-4} \mathrm{eV}^{2}$, suggested a possible experiment. Thus, the effect would be large, but the optimal baseline would be determined by $L / E_{V} \sim 10^{4} \mathrm{~m} / \mathrm{MeV}$. For reactor neutrino disappearance, this implies a distance $L \sim 40 \mathrm{~km}$.

The KamLAND [10] experiment, shown in Fig. 1, was built to test this solution. A key to the design was that the Kamioka mine was (very roughly) equidistant from a large number of Japanese nuclear power plants, and that this distance was many tens of kilometers. Therefore, the first term in brackets in (2.1) dominates and energy resolution washes out the other terms. The detector itself was 1000 tons of liquid scintillator, and protons were used for the delayed neutron capture signal. The IBD event rate was on the order of one per day.

Figure 1 also shows their results. One plots the neutrino disappearance signal as a function of $L / E_{V}$, showing clearly the oscillation pattern represented by the first term in braces of Eq. 2.1. The amplitude of the oscillations give the mixing angle as $\tan ^{2} \theta_{12}=0.481_{-0.080}^{+0.092}$ and the wavelength gives $\delta m_{21}^{2}=\left(7.54_{-0.18}^{+0.19}\right) \times 10^{-5} \mathrm{eV}^{2}$.

By this time, the $\mathrm{K} 2 \mathrm{~K}$ experiment [18] had good evidence for muon neutrino disappearance through neutrino oscillations with a maximal value of $\theta_{23}\left(=45^{\circ}\right)$ and $\delta m_{32}^{2} \approx 2 \times 10^{-3} \mathrm{eV}^{2}$. It so happens that the Chooz experiment [9] was located close to the optimal distance for $\delta m_{31}^{2} \approx$ $\delta m_{32}^{2}$ (since $\delta m_{21}^{2}$ was very small), and their null result implied a limit $\sin ^{2}\left(2 \theta_{13}\right) \leq 0.15$. It was immediately a mystery why $\theta_{23}$ and $\theta_{21}$ were so large, but $\theta_{13}$ appeared to be so small. This put an additional priority on trying to measure $\theta_{13}$, or at least to determine a more stringent upper limit.

An additional motivation to search for a nonzero $\theta_{13}$ came from cosmology. There was a growing theoretical bias that leptogenesis [19] would be responsible for explaining the baryon- 

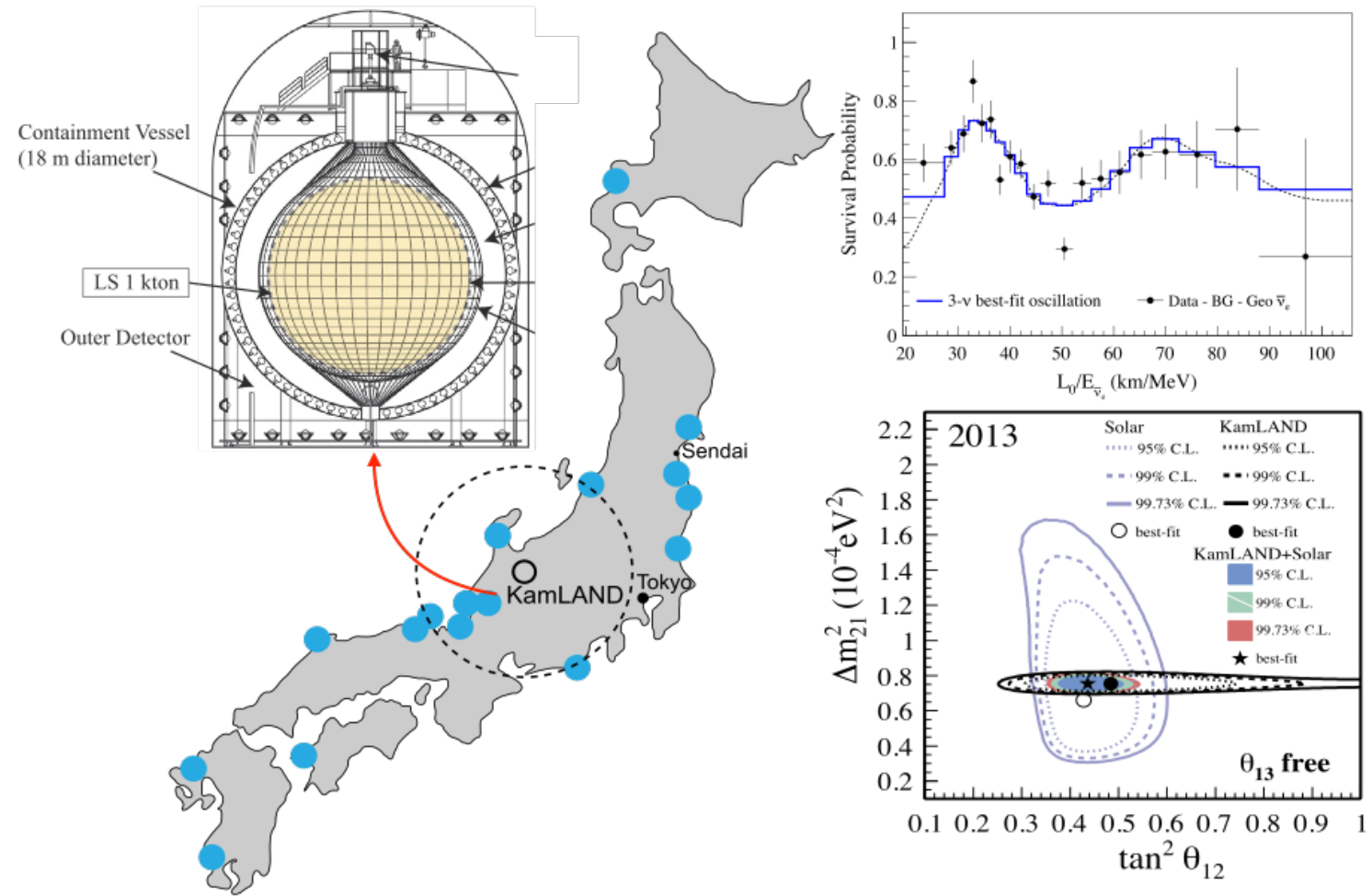

Figure 1: The KamLAND [10] experiment and their results.

antibaryon asymmetry in the universe. This required that there be $C P$ violation in the neutrino sector. If $\theta_{13}=0$, however, then (at least for the known, light neutrinos) the $3 \times 3$ mixing matrix would decouple into $2 \times 2$ matrices and $C P$ violation could not be supported.

By combining KamLAND and solar neutrino data, there were hints [10] of a nonzero $\theta_{13}$ that was just barely consistent with the Chooz results. In the mid-2000's, then, a "race" of sorts was on between three experiments trying to measure $\theta_{13}$.

\section{The $\theta_{13}$ Experiments: Daya Bay, RENO, and Double Chooz}

It is a challenge to measure a small value of $\theta_{13}$ using reactor disappearance. Rewrite (2.1) as

$$
P_{e e}=1-\cos ^{4}\left(\theta_{13}\right) \sin ^{2}\left(2 \theta_{12}\right) \sin ^{2}\left(\left(\Delta_{21}\right)-\sin ^{2}\left(2 \theta_{13}\right) \sin ^{2}\left(\Delta_{e e}\right)\right.
$$

where

$$
\sin ^{2}\left(\Delta_{e e}\right)=\cos ^{2}\left(\theta_{12}\right) \sin ^{2}\left(\Delta_{31}\right)+\sin ^{2}\left(\theta_{12}\right) \sin ^{2}\left(\Delta_{32}\right)
$$

and realize that $\Delta_{31} \approx \Delta_{32} \approx \Delta_{e e}$ since $\Delta_{21}$ is small. Using $\delta m_{32}^{2} \approx 2 \times 10^{-3} \mathrm{eV}$ from accelerator experiments, the optimal distance for a reactor disappearance search (where, again, the mean detected neutrino energy is about $4 \mathrm{MeV}$ ) would be $2 \mathrm{~km}$.

It was quite feasible to mount an experiment at this distance from a nuclear power plant. However, to be sensitive to a small $\theta_{13}$, say $\sin ^{2}\left(2 \theta_{13}\right)$ down to 0.01 , the maximum disappearance probability from (4.1), dominated by the third term, would be only $1 \%$. The uncertainty in any 
calculation of the reactor flux, however, was much larger than that. It would not be possible to put a single detector at kilometer distance and draw any conclusions at this level.

The solution to this challenge was to build multiple detectors and measure the neutrino flux at both a "near" site, much less than $2 \mathrm{~km}$ from the reactor, and also at a "far" site as close to the optimal distance as possible. This way, any deviation of the rate from $1 / r^{2}$ can be detected with precision that was primarily limited by the detectors themselves. In essence, the near detector was a monitor of the reactor flux before oscillation could occur.

Three experiments were eventually mounted, all in the mid-to-late 2000's, based on this approach. One of them, called Double Chooz [20], makes use of the same power plant and detector cave (for the far detector) as did Chooz [9], with a second functionally identical detector planned for the near position. As Chooz was located $1 \mathrm{~km}$ from the reactor core, Double Chooz is not at the optimal location, but should still have good sensitivity to $\theta_{13}$ oscillations.

The RENO experiment [21] was conceived as two new detectors, one near and one far, located along a line that bisected a string of nuclear reactors. Although this geometry has a favorable symmetry, the actual baselines and power fluctuations from the different reactor cores can contribute significantly to the systematic error [22].

The Daya Bay Reactor Neutrino Experiment [23] was the most complex of the three experiment, but also aimed at the highest sensitivity to $\theta_{13}$. Six nuclear reactor cores, for a total peak thermal power of $17 \mathrm{GW}$, were used, along with four detectors in two "near" positions close to the reactors, and four detectors at an optimal "far" location.

All three experiments made use of the surrounding terrain to provide significant overburden for cosmic ray shielding. A nonzero result was first reported by Daya Bay, with results from RENO following close behind. Just prior to this conference, first results with their near detector were released by the Double Chooz collaboration.

This review will use the Daya Bay experiment to illustrate details, but all three experiments are rather similar in their design. The six panels of Figure 2 summarize the experiment. The top left shows the layout of the experiment, including the six cores D1 and D2 (the "Daya Bay" reactors), and $L 1, L 2, L 3$, and $L 4$ (the "Ling Ao" reactors), and the experimental halls. The two "near" halls EH1 and EH2 each support two antineutrino detectors. These four detectors are, essentially, the monitors of the neutrino flux from the six reactor cores. The four detectors in the "far" hall EH3 are at the optimum location (i.e. $\approx 2 \mathrm{~km}$ ) to observe $\bar{v}_{e}$ disappearance due to a nonzero $\theta_{13}$. The halls are under various amounts of rock overburden, with the largest amount over EH3.

The eight antineutrino detectors ("AD's") are functionally identical, a so-called "three zone" design, as shown on the upper right of Figure 2. An inner acrylic vessel, $3 \mathrm{~m}$ diameter and $3 \mathrm{~m}$ high, contains the 20 ton target of gadolinium loaded liquid scintillator. An outer $4 \mathrm{~m}$ acrylic vessel contains unloaded liquid scintillator, and aims to collected gamma rays from $n$-Gd capture for events near the outer boundary of the inner vessel. A total of 192 photomultipliers (PMTs) are arranged on the cylinder of the $5 \mathrm{~m}$ stainless steel vessel, which is filled with mineral oil as passive shielding against ambient radioactive backgrounds from the PMTs. Three automatic calibration units (ACUs) sit atop each detector, and the entire assembly sits in a $10 \mathrm{~m}$ deep pool of high purity water. This provides more shielding from radioactive backgrounds, and is also instrumented with PMTs which are an active detector of cosmic ray muons which produce Čerenkov light in the water. See $[24,25,26,27]$ for complete technical information on the Daya Bay experiment. 

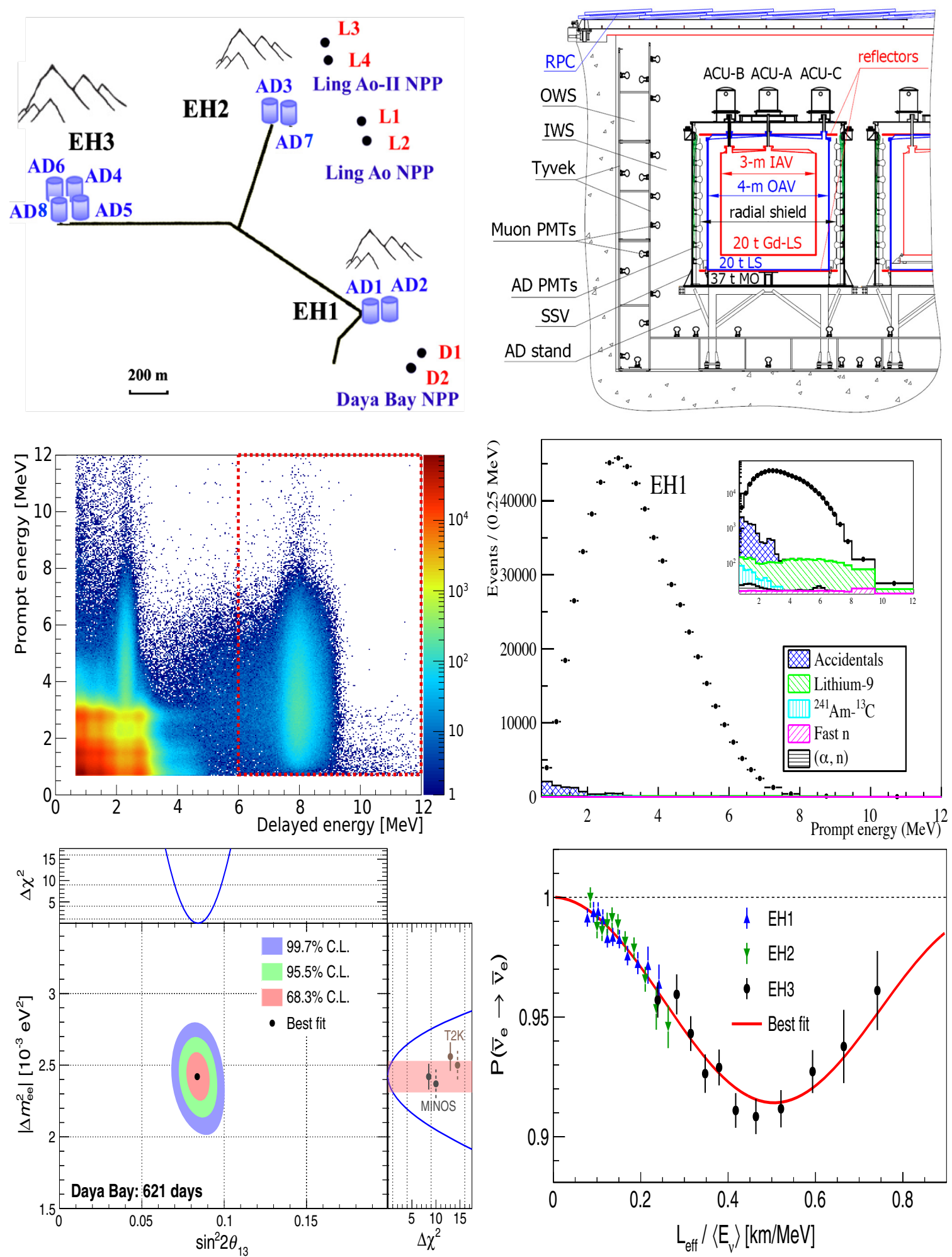

Figure 2: The Daya Bay Reactor Neutrino Experiment, including the layout, cutaway of an antineutrino detector, prompt versus delayed energy signals, prompt energy for IBD events, $\chi^{2}$ contours for the best fit to $\theta_{13}$ and $\Delta m_{e e}^{2}$, and the disappearance probability. See text for details. 
The middle two panels of Figure 2 show Daya Bay energy spectra in the ADs. Recall from Section 1 that Reaction 1.1 gives rise to two signals. One is the "prompt" energy from the positron kinetic energy plus the the annihilation gamma rays. This prompt energy equals the neutrino energy minus $0.78 \mathrm{MeV}$. The second signal is from delayed neutron capture on gadolinium, which follows in tens of microseconds and releases $\sim 8 \mathrm{MeV}$ of gamma rays. The first panel clearly shows the signal region (enclosed in a red box) which is virtually free of background. The second panel shows the projection onto the prompt energy in the signal region. Backgrounds components are labeled, and seen in the insert. The scatterplot also shows the $2.2 \mathrm{MeV}$ gamma rays from $n p$ capture and the large residual background below $3 \mathrm{MeV}$ giving accidental coincidences.

Neutrino oscillations will distort the spectrum shape for ADs in EH3 relative to those in EH1 and $\mathrm{EH} 2$, regardless of the shape of the reactor spectrum. We perform a fit that incorporates Equation 4.1 into a simultaneous description of all detectors, including the distortion of the spectrum in the EH1 ADs from the Ling Ao reactors, and vice versa. This leaves some residual systematic uncertainty due to the reactor spectrum shape, but it is small.

The result of this fit, using the full detector configuration [11], is shown in the lower left panel of Figure 2. Combining statistical and systematic errors, Daya Bay finds ${ }^{1}$

$$
\sin ^{2} 2 \theta_{13}=0.085 \pm 0.005 \quad\left|\Delta m_{e e}^{2}\right|=(2.42 \pm 0.11) \times 10^{-3} \mathrm{eV}^{2} \quad \text { (Daya Bay) }
$$

The plot also indicates the values of $\Delta m_{23}^{2}$ as determined from T2K [28] and MINOS [29] using both normal and inverted mass hierarchy.

The lower right panel of Figure 2, used for illustration only and does not detail the fitting procedure, plots the disappearance probability (constrained to be unity at zero distance from the reactor) as a function of an effective $L / E$, for spectral data measured in each of the three halls. The red line shows Equation 4.1 using the best fit parameters.

Such a large value of $\theta_{13}$ was quite a surprise [30]. It is just below the upper limit originally published by Chooz [9], although there had been some evidence for a nonzero $\theta_{13}$ from solar neutrinos [31], T2K [32], and from Double Chooz using a single detector in their far hall [33]. Then, soon after Daya Bay published the first measurement with both near and far detectors, RENO confirmed this [34] and it was clear that $\theta_{13}$ oscillations were not such a small effect after all.

RENO has recently published [12] a higher statistics update of their first analysis, including a spectral measurement therefore giving a result for $\Delta m_{e e}^{2}$. Their analysis is similar to that used by Daya Bay, with results shown in Figure 3. They find

$$
\begin{aligned}
\sin ^{2} 2 \theta_{13} & =0.082 \pm 0.009 \text { (stat) } \pm 0.006 \text { (syst) } \\
\left|\Delta m_{e e}^{2}\right| & =\left(2.62_{-0.23}^{+0.21}(\text { stat })_{-0.13}^{+0.12} \text { (syst) }\right) \times 10^{-3} \mathrm{eV}^{2}
\end{aligned}
$$

The Double Chooz collaboration has recently released their first results using both near and far detectors [13]. With detectors at $0.4 \mathrm{~km}$ and $1.1 \mathrm{~km}$, they find

$$
\sin ^{2} 2 \theta_{13}=0.111 \pm 0.018 \quad \text { (Double Chooz) }
$$

\footnotetext{
${ }^{1}$ After this presentation at FPCP 2016, but before these proceedings, the Daya Bay collaboration released results from an analysis of a larger data set for Neutrino 2016. Our current result is

$$
\sin ^{2} 2 \theta_{13}=0.0841 \pm 0.0027 \text { (stat) } \pm 0.0019 \text { (syst) } \quad\left|\Delta m_{e e}^{2}\right|=(2.50 \pm 0.06 \text { (stat) } \pm 0.06 \text { (syst) }) \times 10^{-3} \mathrm{eV}^{2}
$$
}



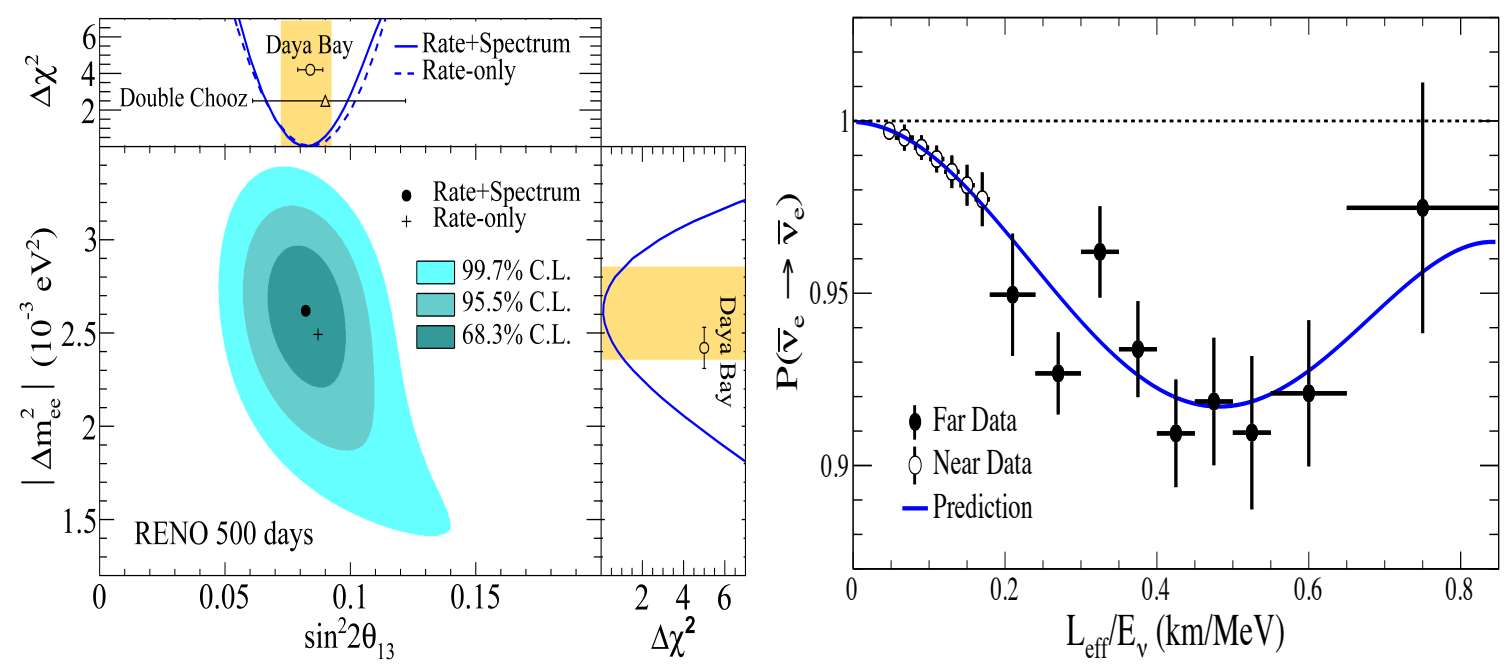

Figure 3: Results from the latest RENO analysis [12] . See text for details.

\section{Reactor Neutrino Spectra and "The Bump"}

The new generation of $\theta_{13}$ experiments, Daya Bay, RENO, and Double Chooz, have collected, and will continue to collect, millions of IBD events. This means that the underlying neutrino spectrum from nuclear power reactors can now be determined with unprecedented precision.

Understanding this spectrum has more than just an academic interest. For Daya Bay, for example, knowledge of the reactor spectrum contributes significantly to the systematic error. From the perspective of nuclear engineering, the underlying $\beta^{-}$decays contribute several percent to the reactor thermal power. Details of the reactor spectrum informs details of the relevant nuclear data bases for fission fragment distributions and beta decays of those fragments. Furthermore, by studying specific details of the neutrino spectra, we can learn about reactor fuel burn-up rates and the time evolution of the underlying ${ }^{235} \mathrm{U},{ }^{239} \mathrm{Pu},{ }^{241} \mathrm{Pu}$, and ${ }^{238} \mathrm{U}$ components.

Figure 4 shows reactor IBD spectrum results from Data Bay [35], RENO [36], and Double Chooz [13]. All three experiments show a feature that was entirely unexpected, namely an excess of events in the region near $5 \mathrm{MeV}$. This excess, often referred to as a "bump" or "shoulder", is apparently inconsistent with any reasonable "new physics" hypotheses, but instead appears to be an artifact of the neutrino spectrum itself. Explanations for this feature [5, 37, 38, 39, 40] point back to details of the fission fragment distributions and beta decay spectra germane to power reactors.

One intriguing possibility is that the "bump" is from the ${ }^{238} \mathrm{U}$ fast-neutron fission component [5, 38]. This is particularly attractive since experiments [41, 42, 43, 44, 45] that measured the electron spectra from thermal-neutron induced fission fragments of ${ }^{235} \mathrm{U},{ }^{239} \mathrm{Pu}$, and ${ }^{241} \mathrm{Pu}$ showed no evidence of this feature. A recent measurement [46], however, of the beta spectrum from ${ }^{238} \mathrm{U}$ fast-neutron fission shows no such feature.

Studies continue regarding the uncertainties associated with calculating reactor neutrino spectra, and experiments are planned that will hopefully shed some light on this issue. Some of these efforts are detailed below, as well as other implications based on comparison of neutrino spectrum calculations with existing measurements. 

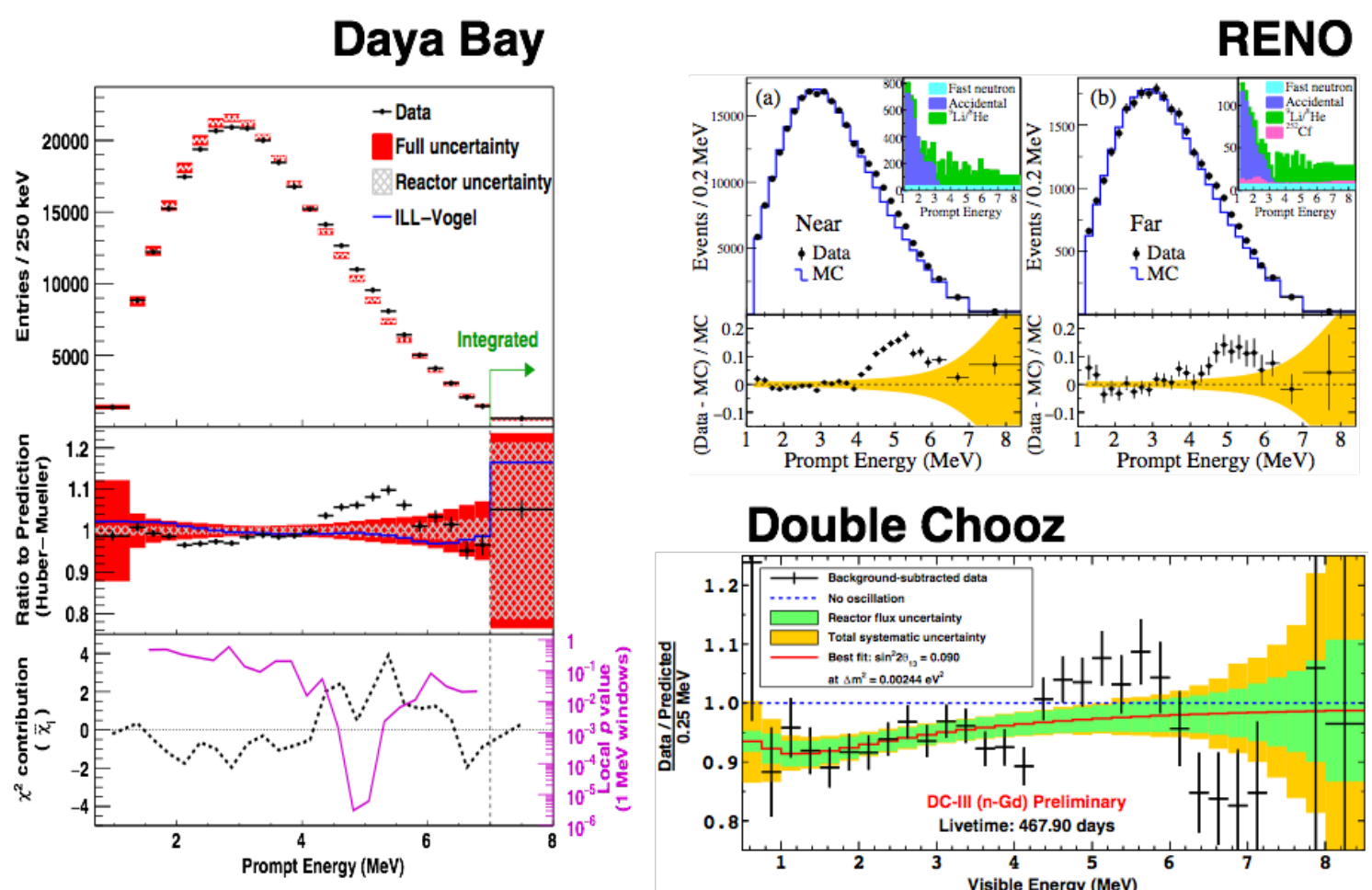

\section{Double Chooz}

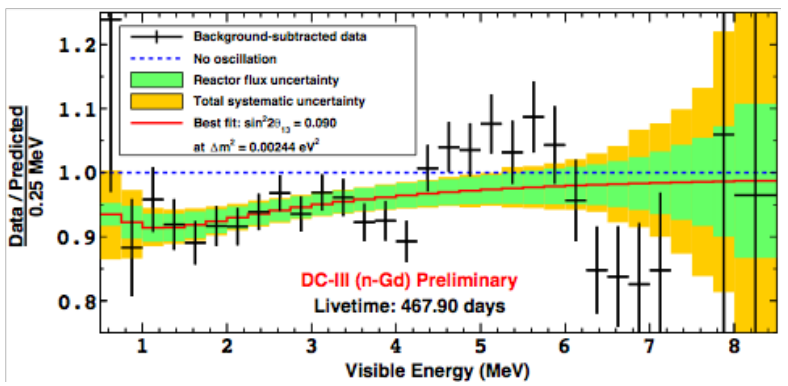

Figure 4: High statistics IBD spectra from Daya Bay and RENO, including comparison to a nominal reactor neutrino spectrum model, and the comparison alone for Double Chooz. A "bump" or "shoulder" is evident in the region of $5 \mathrm{MeV}$ prompt energy.

\section{The Reactor Neutrino Anomaly and Sterile Neutrinos}

Regardless of the level of our knowledge of reactor neutrino spectra, it is of course possible to compare the total flux measured to that predicted.

A recent careful comparison [47] of existing data with the best available calculations, indeed suggests a $6 \%$ deficit in the observed spectra for a wide variety of experiments. This deficit has been dubbed "The Reactor Neutrino Anomaly" and may be an indicator of new physics, namely the existence of "sterile" neutrinos. See, for example, [48] and also A. deGouvea, these proceedings. On the other hand, there are serious uncertainties in the total flux calculation, and there are questions $[49,50]$ concerning the claim that the flux can be in fact calculated to better than $6 \%$ precision.

In conjunction with their spectral measurement, Daya Bay has carried out an analysis of the observed integrated neutrino flux, with careful attention to all uncertainties [35]. The left panel of Figure 5 illustrates the neutrino yield, that is the IBD cross section folded with the reactor spectrum, for three of the near ADs and three of the far ADs. The error bars are statistical, and the gray band indicates the overall systematic uncertainty. These measurements are compared to two calculations. The ILL-Vogel model (in orange) refers to the conventional ILL model [41, 42, 43, 44] of ${ }^{235} \mathrm{U}$, ${ }^{239} \mathrm{Pu}$, and ${ }^{241} \mathrm{Pu}$, and the theoretical model of ${ }^{238} \mathrm{U}$ from Vogel [51]. The Huber- Mueller model refers to the recent reevaluation of ${ }^{235} \mathrm{U},{ }^{239} \mathrm{Pu}$, and ${ }^{241} \mathrm{Pu}$ from Huber [52], and that of ${ }^{238} \mathrm{U}$ from 

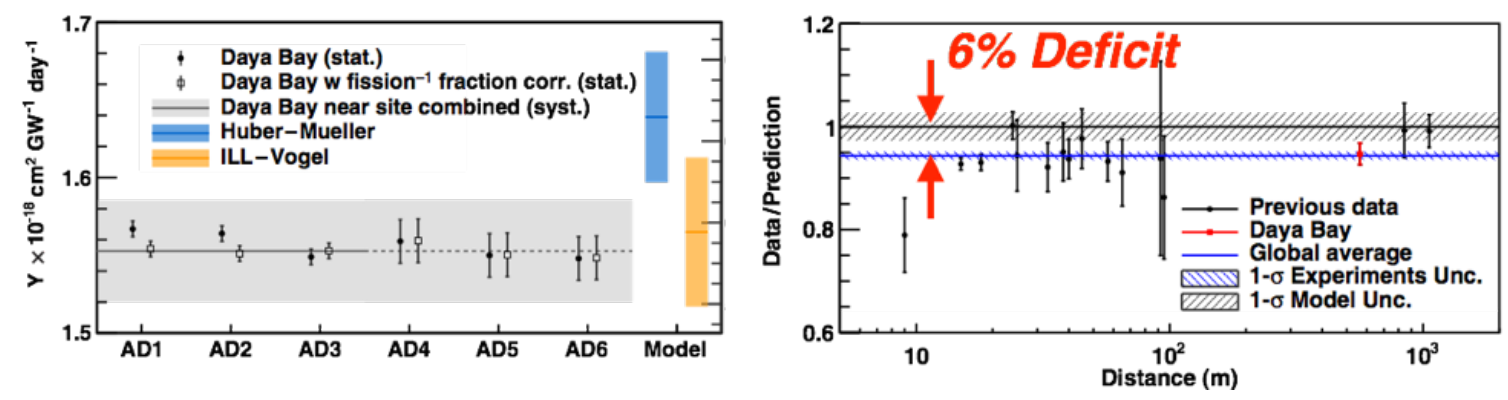

Figure 5: Illustration of the Reactor Neutrino Anomaly, from Daya Bay [35]. See also [47] for the original version of the figure on the right.

Mueller et al. [53]. Clearly, the anomaly appears with the more up to date calculation, but Hayes, et al. [49] argue that the systematic error bands are too small on all these calculations.

The right hand panel of Figure 5 is an updated version of Figure 5 from [47] with the Daya Bay result [35] added. There is indeed an across-the-board deficit over all distances. If this is to be interpreted in terms of sterile neutrinos, then the oscillation length must be very short, on the order of meters or less. This implies a value of $\Delta m^{2}$ on the order of $1 \mathrm{eV}^{2}$.

Global fits $[54,55]$ have been performed, to the reactor anomaly and including other potentially consistent measurements, including calibrations for gallium solar neutrino experiments [56]. One rendition of these analyses, taken from [57], is shown in Figure 6. Although there is some tension between the gallium and reactor experiments, there is significant overlap with a preference for a fourth generation sterile neutrino with $\Delta m^{2} \approx 2 \mathrm{eV}^{2}$ and $\sin ^{2} 2 \theta \approx 0.1$.

The implications for reactor neutrino experiments is obvious. One clearly wants to perform a $\bar{v}_{e}$ disappearance experiment with baseline on the order of meters.

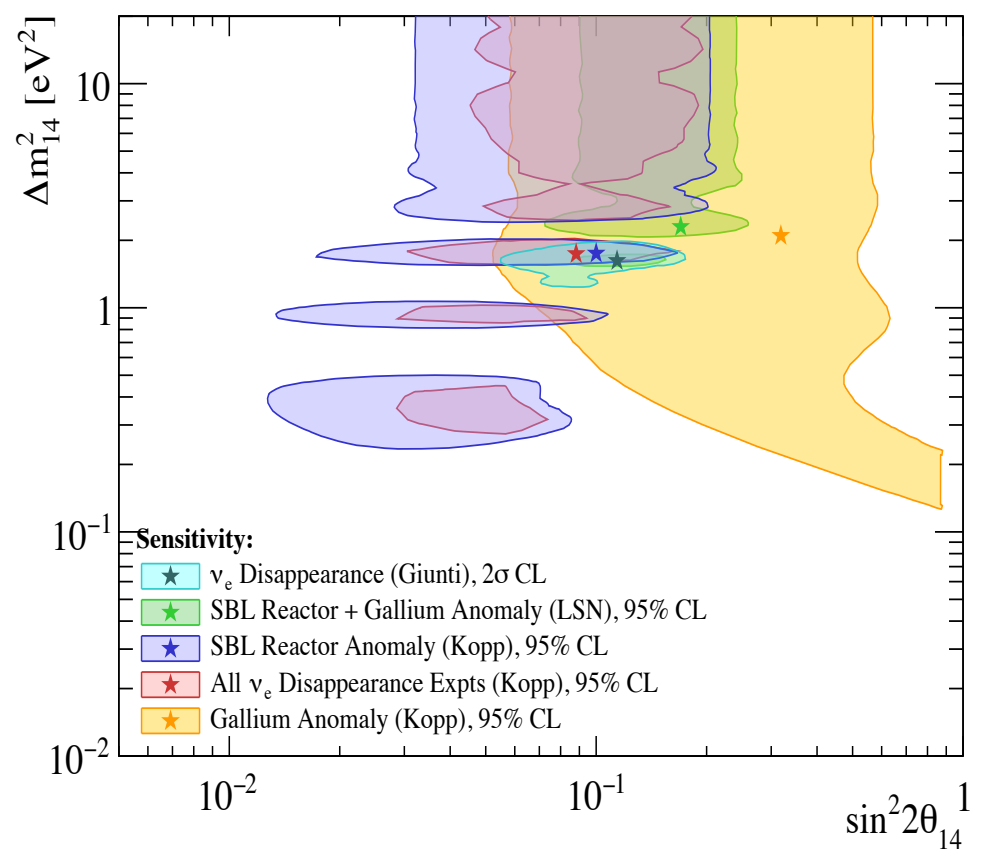

Figure 6: Allowed region for mass and mixing parameters for a sterile neutrino from $\bar{v}_{e}$ disappearance experiments at reactors and in the calibration of gallium solar neutrino experiments. See the text for references and details. 


\section{Very Short Baseline Reactor Experiments}

There are challenges to observing $\bar{v}_{e}$ disappearance oscillations at baselines on the order of meters, that were not faced by the $\theta_{13}$ experiments. Heeger, et al., [58], outline these challenges and the options for dealing with them. Research reactors are the source of choice, rather than nuclear power plants. The core sizes are much more compact, and it is feasible to mount a detector within several meters of them. The power of a research reactor is one to two orders of magnitude smaller than a power reactor, but the distance is much closer so these factors more or less cancel out. On the other hand, backgrounds associated with the reactor itself are now an issue, and cosmic ray shielding is a greater challenge because overburden is difficult to achieve.

Another issue is that to observe the oscillations themselves, the detector must be segmented longitudinally so that spectral modifications can be made as a function of distance. This segmentation is in fact a handle on dealing with backgrounds.

Of course, such an experiment will provide a careful measurement of the underlying reactor neutrino spectrum. Furthermore, since research reactors typically make use of highly enriched ${ }^{235} \mathrm{U}$ cores, fuel evolution and contributions from ${ }^{239} \mathrm{Pu}$, and ${ }^{241} \mathrm{Pu}$ are no longer an issue.

Several "Very Short Baseline" (VSBL) oscillation experiments are being mounted at research reactors worldwide, as well as a few efforts (under special circumstances) at nuclear power plants. The presentation focussed on the Precision Oscillation and Spectrum experiment (PROSPECT) [57, 59] based at the High Flux Isotope Reactor (HFIR) at Oak Ridge National Laboratory (ORNL) in the US; the Short baseline Oscillation search with Lithium-6 Detector (SoLiD) [60] located at the Belgian Reactor 2 (BR2) at the Belgian Nuclear Research Centre (SCK-CEN) in Belgium; and the STEREO experiment [61] at the research reactor at the Institute Laue Langevin (ILL) in France.

Based at research reactors, each experiment to take data with the reactor completely turned off, so that cosmic ray backgrounds can be separated from reactor-related backgrounds. Detector segmentation is further useful so that fiducial volumes can be identified, again facilitating background rejection. A study has been performed [62] by the PROSPECT collaboration to identify and categorize the backgrounds at different research reactors.

Although all aiming at essentially the same physics, the details of all the VSBL experiments vary quite a bit. For example, although STERO uses $\mathrm{Gd}$ for the neutron capture signal, both PROSPECT and SoLiD make use of the reaction ${ }^{6} \mathrm{Li}+n \rightarrow{ }^{4} \mathrm{He}+{ }^{3} \mathrm{H}$, which produces a highly localized signal at the expense of visibly deposited energy. In all cases, though, the object is to measure the $\bar{v}_{e}$ IBD spectrum as a function of distance with a position resolution on the order of $20 \mathrm{~cm}$, commensurate with the size of the research reactor core.

The sensitivities of these experiments are also similar. Figure 7 shows the sensitivity for PROSPECT. This detector is an array of one-dimensional cells, $1.2 \mathrm{~m}$ long and $15 \times 15 \mathrm{~cm}^{2}$, filled with ${ }^{6} \mathrm{Li}$-doped liquid scintillator with hemispherical photomultiplier tubes on each end. The experiment envisions a second phase with a larger detector at a greater distance which affords a greater sensitivity, but the Phase I detector is movable and thereby achieves a reach which includes the best fit parameters for sterile neutrinos.

A different but noteworthy approach to very short baseline $\bar{v}_{e}$ disappearance is the DANSS [63] experiment. ${ }^{2}$ This collaboration places a highly segmented solid scintillator detector, with modules

\footnotetext{
${ }^{2}$ I did not discuss DANSS in my conference presentation.
} 

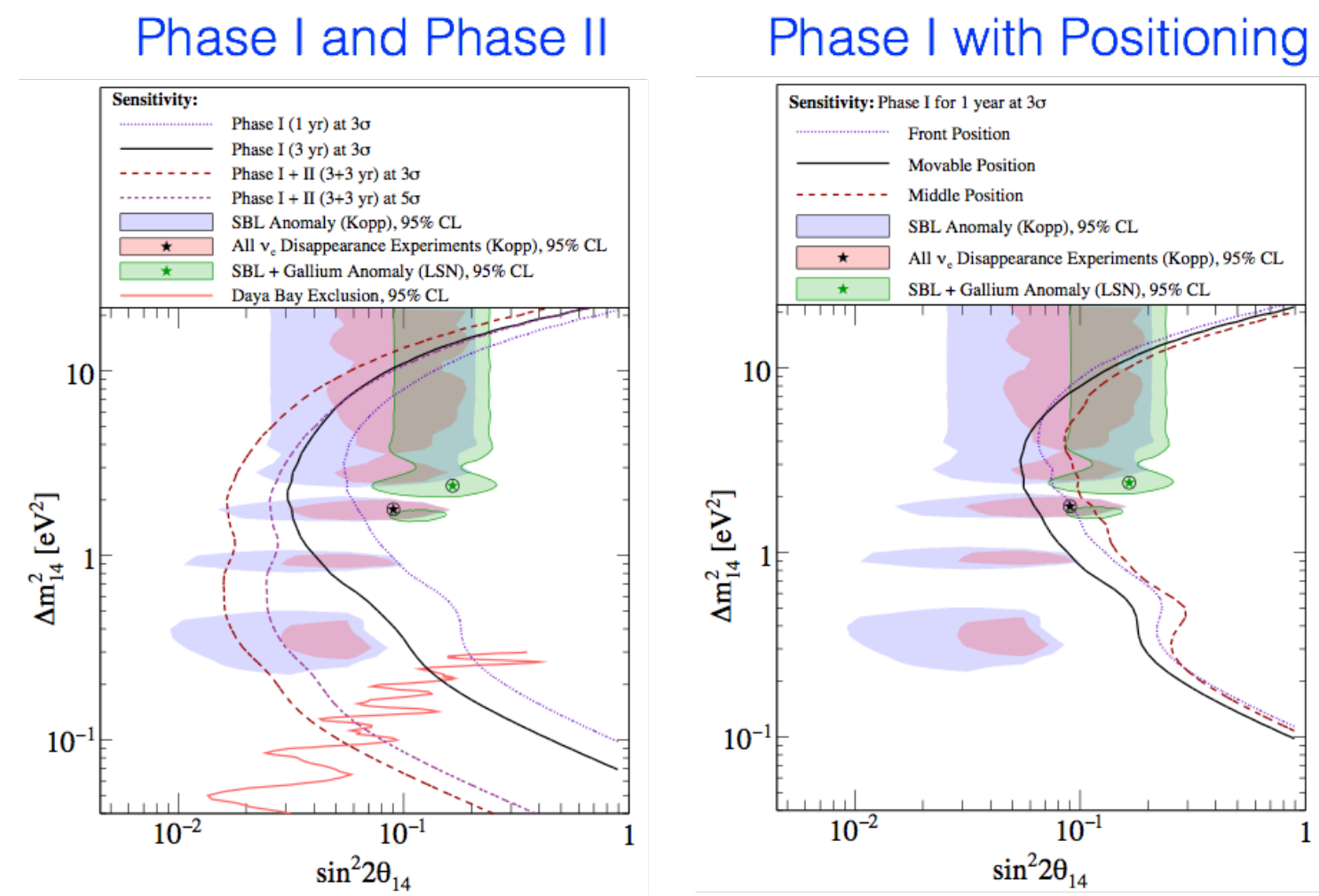

Figure 7: Sensitivity to sterile neutrino oscillation parameters for PROSPECT [57] and comparison to the global best fit parameters in Figure 6.

that include a coating of plastic infused with gadolinium oxide for neutron detection. The apparatus sits directly beneath the core of a $\sim 3 \mathrm{GW}$ power reactor, so the IBD rate is very large. The reactor and containment vessel provide significant cosmic ray shielding, providing a reasonable signal to background ratio. The active volume of the detector is $\approx 1 \mathrm{~m}^{3}$, with a granularity of a few $\mathrm{cm}$. It sits on a platform that moves vertically, allowing the distance between the centers of the detector and the reactor core to vary between $10 \mathrm{~m}$ and $12 \mathrm{~m}$. The DANSS collaboration started taking regular data in April 2016 and first results are expected shortly.

\section{Mass Hierarchy and the JUNO Experiment}

Learned, et al. [8], realized something novel about Equation 2.1 which allows one to measure the neutrino mass hierarchy using $\bar{v}_{e}$ disappearance. For normal hierarchy,

$$
\left|\delta m_{31}^{2}\right|=\left|\delta m_{32}^{2}\right|+\left|\delta m_{21}^{2}\right| \quad \text { so } \quad\left|\delta m_{31}^{2}\right|>\left|\delta m_{32}^{2}\right|
$$

whereas for inverted hierarchy,

$$
\left|\delta m_{31}^{2}\right|=\left|\delta m_{32}^{2}\right|-\left|\delta m_{21}^{2}\right| \quad \text { so } \quad\left|\delta m_{31}^{2}\right|<\left|\delta m_{32}^{2}\right|
$$

This amounts to $\mathrm{a} \approx 3 \%$ difference in the wavelength of the $\theta_{13}$ oscillations after interference with the $\theta_{12}$ oscillations. Consequently, a KamLAND-like experiment at large distance from a strong source of $\bar{v}_{e}$, but having excellent energy resolution, can in principle determine the mass hierarchy. 

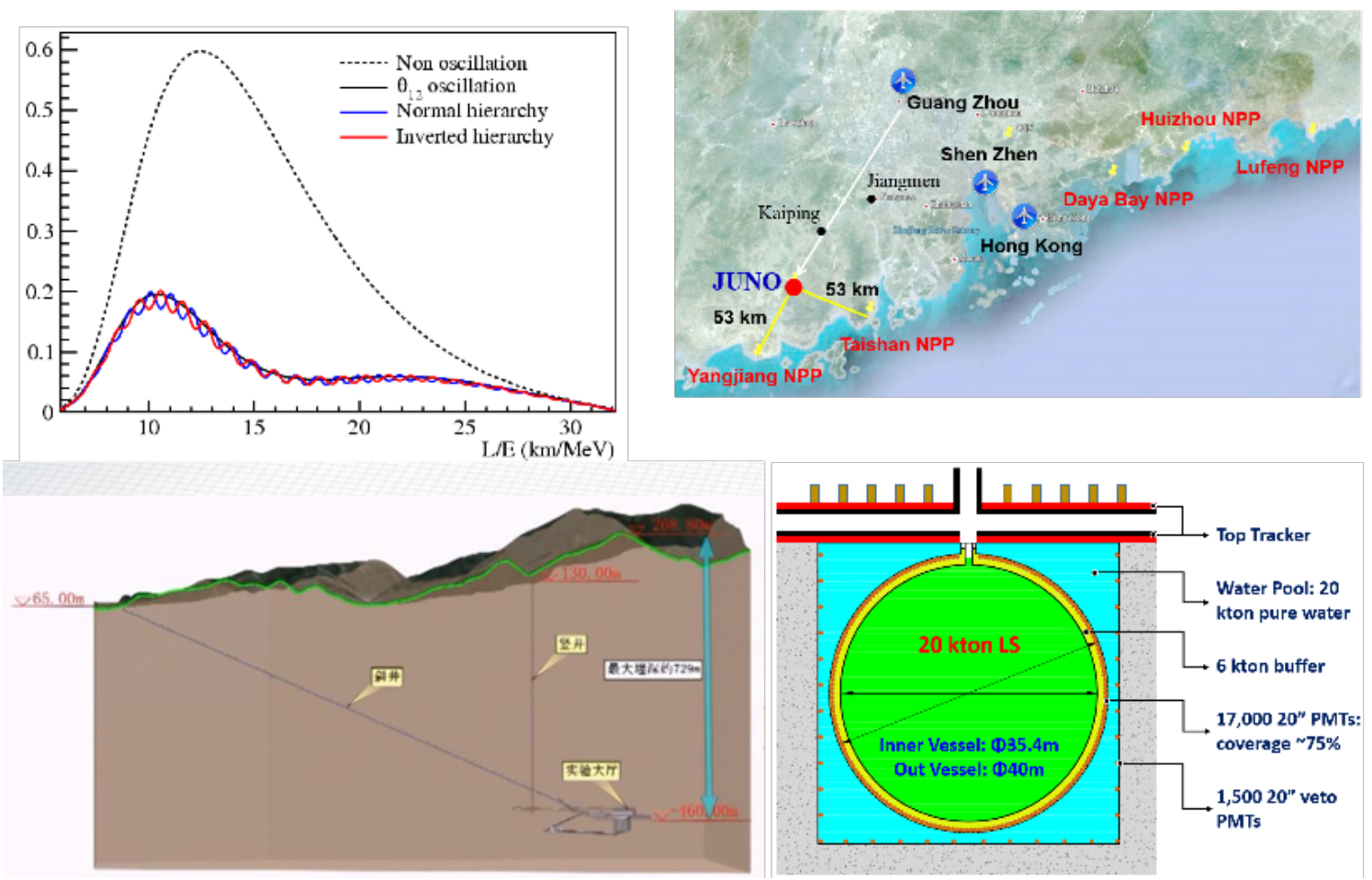

Figure 8: Physics concept for measuring the neutrino mass hierarchy using Equation 2.1 and the JUNO experiment $[14,64,65]$. Other expected physics results include a precise measurement of $\theta_{12}$, astrophysical supernova detection, and neutrinos from the Earth's core.

This is the goal of the Jiangmen Underground Neutrino Observatory (JUNO), currently under construction in southeastern China [14, 64, 65]. Figure 8 illustrates the concept of different frequencies for the $\theta_{13} / \theta_{12}$ interference term in $\bar{v}_{e}$ disappearance, shows the location of JUNO relative to nearby nuclear power plants (and Hong Kong and Daya Bay), and includes schematics of the underground laboratory and large liquid scintillator antineutrino detector.

The detector is located at a new site, equidistant $(53 \mathrm{~km})$ from two large nuclear power plants in Yangjian and Tiashan, totaling $27 \mathrm{GW}$ thermal total power. This is an optimum distance for $\theta_{12}$ oscillations, maximizing the effect shown on the upper left panel, plotting the $\theta_{13} / \theta_{12}$ interference effect as a function of $L / E$, for the two different mass hierarchy cases. The vertical scale is arbitrary, but shows the large suppression relative to "non oscillation" from $\theta_{12}$ disappearance, and also the "wiggles" from $\theta_{13}$ disappearance.

The detector itself sits $600 \mathrm{~m}$ underground, accessible by inclined and vertical tunnels from the surface, and consists of a 20 kton liquid scintillator sphere, viewed by 17,000 large photomultiplier tubes, providing very good energy resolution. The sphere is submerged in a water pool, instrumented with photomultipliers itself, similar to the active shield employed in Daya Bay. Delayed neutrons from IBD events will be identified using the $n p$ capture process on protons in the scintillator itself. Precision energy calibration schemes will be in place.

Civil construction has begun, and is expected to be completed in 2017. Detector components are currently being prepared, and the detector installation is planned for 2018-2019. The liquid filling campaign and start of data taking will begin in 2020 . 


\section{Conclusions and Acknowledgements}

Neutrino experiments at nuclear reactors remain critical contributors to fundamental physics. Nuclear power plants are strong sources but not so well understood, so "near detectors" are crucial for carrying out precision measurements of the disappearance amplitude. Research reactors are not so powerful, but offer more control over interpretation of results, and we expect new results soon on searches for sterile neutrinos from such facilities. Within the next five year or so, we should also be seeing results from JUNO on the mass hierarchy question, as well as other physics topics.

Many thanks to Dave Hitlin and Caltech for putting together an outstanding conference. I also acknowledge the many Caltech faculty, staff, and graduates who have contributed to reactor neutrino physics over the years, including Felix Boehm, Andreas Piepke, Petr Vogel, John Beacom, Bob McKeown, Brian Fujikawa, Chao Zhang, Raymond Tsang, Xin Qian, Jianglai Liu, Dan Dwyer, Glenn Horton-Smith, Christopher Mauger, Pedro Ochoa-Ricoux, and Michael Mendenhall.

\section{References}

[1] F. Reines and C. L. Cowan, "Detection of the free neutrino," Phys. Rev. 92, 830 (1953).

[2] C. L. Cowan, F. Reines, F. B. Harrison, H. W. Kruse and A. D. McGuire, "Detection of the free neutrino: A Confirmation," Science 124, 103 (1956).

[3] F. Reines and C. L. Cowan, "Free anti-neutrino absorption cross-section. 1: Measurement of the free anti-neutrino absorption cross-section by protons," Phys. Rev. 113, 273 (1959).

[4] F. Reines, C. L. Cowan, F. B. Harrison, A. D. McGuire and H. W. Kruse, "Detection of the free anti-neutrino," Phys. Rev. 117, 159 (1960).

[5] A. C. Hayes and P. Vogel, "Reactor Neutrino Spectra," arXiv:1605.02047 [hep-ph].

[6] P. Vogel and J. F. Beacom, "Angular distribution of neutron inverse beta decay, $v_{e}+p \rightarrow e^{+}+n$," Phys. Rev. D 60, 053003 (1999)

[7] G. Zacek et al. [CALTECH-SIN-TUM Collaboration], "Neutrino Oscillation Experiments at the Gosgen Nuclear Power Reactor,” Phys. Rev. D 34, 2621 (1986).

[8] J. Learned, S. T. Dye, S. Pakvasa and R. C. Svoboda, "Determination of neutrino mass hierarchy and $\theta_{13}$ with a remote detector of reactor antineutrinos," Phys. Rev. D 78, 071302 (2008)

[9] M. Apollonio et al. [CHOOZ Collaboration], "Search for neutrino oscillations on a long baseline at the CHOOZ nuclear power station,” Eur. Phys. J. C 27, 331 (2003)

[10] M. P. Decowski [KamLAND Collaboration], "KamLAND's precision neutrino oscillation measurements,” Nucl. Phys. B 908, 52 (2016).

[11] F. P. An et al. [Daya Bay Collaboration], "New Measurement of Antineutrino Oscillation with the Full Detector Configuration at Daya Bay,” Phys. Rev. Lett. 115, no. 11, 111802 (2015)

[12] J. H. Choi et al. [RENO Collaboration], "Observation of Energy and Baseline Dependent Reactor Antineutrino Disappearance in the RENO Experiment," Phys. Rev. Lett. 116, no. 21, 211801 (2016)

[13] R. Carr, S. Lutch and P. Novella, "New results from the Double Chooz experiment," Nucl. Part. Phys. Proc. 273-275, 2648 (2016).

See also http://theory.fnal.gov/jetp/talks/DCIV@FNAL_Anatael_160315.pdf 
[14] Z. Djurcic et al. [JUNO Collaboration], “JUNO Conceptual Design Report," arXiv:1508.07166 [physics.ins-det].

[15] A. B. McDonald, "The Sudbury Neutrino Observatory: Observation of flavor change for solar neutrinos," Annalen Phys. 528, 469 (2016).

[16] S. P. Mikheev and A. Y. Smirnov, "Resonance Amplification of Oscillations in Matter and Spectroscopy of Solar Neutrinos,” Sov. J. Nucl. Phys. 42, 913 (1985) [Yad. Fiz. 42, 1441 (1985)].

[17] L. Wolfenstein, “Neutrino Oscillations in Matter,” Phys. Rev. D 17, 2369 (1978).

[18] M. H. Ahn et al. [K2K Collaboration], "Indications of neutrino oscillation in a $250 \mathrm{~km}$ long baseline experiment,” Phys. Rev. Lett. 90, 041801 (2003)

[19] S. Davidson, E. Nardi and Y. Nir, "Leptogenesis,” Phys. Rept. 466, 105 (2008)

[20] F. Ardellier et al. [Double Chooz Collaboration], "Double Chooz: A Search for the neutrino mixing angle $\theta_{13}$, , hep-ex/0606025.

[21] J. K. Ahn et al. [RENO Collaboration], "RENO: An Experiment for Neutrino Oscillation Parameter $\theta_{13}$ Using Reactor Neutrinos at Yonggwang," arXiv:1003.1391 [hep-ex].

[22] A. Cucoanes, P. Novella, A. Cabrera, M. Fallot, A. Onillon, M. Obolensky and F. Yermia, "Reactor Neutrino Flux Uncertainty Suppression on Multiple Detector Experiments,” arXiv:1501.00356 [hep-ex].

[23] X. Guo et al. [Daya Bay Collaboration], "A Precision measurement of the neutrino mixing angle $\theta_{13}$ using reactor antineutrinos at Daya-Bay," hep-ex/0701029.

[24] F. P. An et al. [Daya Bay Collaboration], “The Detector System of The Daya Bay Reactor Neutrino Experiment,” Nucl. Instrum. Meth. A 811, 133 (2016)

[25] F. P. An et al. [Daya Bay Collaboration], "The muon system of the Daya Bay Reactor antineutrino experiment,” Nucl. Instrum. Meth. A 773, 8 (2015)

[26] J. Wilhelmi et al., "The Water Purification System for the Daya Bay Reactor Neutrino Experiment," arXiv:1408.1302 [physics.ins-det], Journal of Water Process Engineering, 5(2015)127

[27] F. P. An et al. [Daya Bay Collaboration], "A side-by-side comparison of Daya Bay antineutrino detectors," Nucl. Instrum. Meth. A 685, 78 (2012)

[28] K. Abe et al. [T2K Collaboration], "Precise Measurement of the Neutrino Mixing Parameter $\theta_{23}$ from Muon Neutrino Disappearance in an Off-Axis Beam,” Phys. Rev. Lett. 112, no. 18, 181801 (2014)

[29] P. Adamson et al. [MINOS Collaboration], "Combined analysis of $v_{\mu}$ disappearance and $v_{\mu} \rightarrow v_{e}$ appearance in MINOS using accelerator and atmospheric neutrinos," Phys. Rev. Lett. 112, 191801 (2014)

[30] F. P. An et al. [Daya Bay Collaboration], "Observation of electron-antineutrino disappearance at Daya Bay,” Phys. Rev. Lett. 108, 171803 (2012)

[31] G. L. Fogli, E. Lisi, A. Marrone, A. Palazzo and A. M. Rotunno, "Evidence of $\theta_{13}>0$ from global neutrino data analysis," Phys. Rev. D 84, 053007 (2011)

[32] K. Abe et al. [T2K Collaboration], "Indication of Electron Neutrino Appearance from an Accelerator-produced Off-axis Muon Neutrino Beam,” Phys. Rev. Lett. 107, 041801 (2011)

[33] Y. Abe et al. [Double Chooz Collaboration], "Indication for the disappearance of reactor electron antineutrinos in the Double Chooz experiment,” Phys. Rev. Lett. 108, 131801 (2012) 
[34] J. K. Ahn et al. [RENO Collaboration], "Observation of Reactor Electron Antineutrino Disappearance in the RENO Experiment,” Phys. Rev. Lett. 108, 191802 (2012)

[35] F. P. An et al. [Daya Bay Collaboration], "Measurement of the Reactor Antineutrino Flux and Spectrum at Daya Bay,” Phys. Rev. Lett. 116, no. 6, 061801 (2016)

[36] S. H. Seo [RENO Collaboration], "New Results from RENO and The $5 \mathrm{MeV}$ Excess," AIP Conf. Proc. 1666, 080002 (2015) doi:10.1063/1.4915563 [arXiv:1410.7987 [hep-ex]].

[37] D. A. Dwyer and T. J. Langford, "Spectral Structure of Electron Antineutrinos from Nuclear Reactors,” Phys. Rev. Lett. 114, no. 1, 012502 (2015) doi:10.1103/PhysRevLett.114.012502

[38] A. C. Hayes, J. L. Friar, G. T. Garvey, D. Ibeling, G. Jungman, T. Kawano and R. W. Mills, "Possible origins and implications of the shoulder in reactor neutrino spectra," Phys. Rev. D 92, no. 3, 033015 (2015)

[39] A. A. Zakari-Issoufou et al. [IGISOL Collaboration], "Total Absorption Spectroscopy Study of ${ }^{92} \mathrm{Rb}$ Decay: A Major Contributor to Reactor Antineutrino Spectrum Shape,” Phys. Rev. Lett. 115, no. 10, 102503 (2015)

[40] C. Buck, A. P. Collin, J. Haser and M. Lindner, "Investigating the Spectral Anomaly with Different Reactor Antineutrino Experiments," arXiv:1512.06656 [hep-ex].

[41] K. Schreckenbach, H. R. Faust, F. von Feilitzsch, A. A. Hahn, K. Hawerkamp and J. L. Vuilleumier, "Absolute measurement of the beta spectrum from ${ }^{235} \mathrm{U}$ fission as a basis for reactor antineutrino experiments,” Phys. Lett. B 99, 251 (1981).

[42] F. Von Feilitzsch, A. A. Hahn and K. Schreckenbach, "Experimental Beta Spectra From ${ }^{239}$ Pu And ${ }^{235}$ U Thermal Neutron Fission Products And Their Correlated Anti-neutrinos Spectra," Phys. Lett. B 118, 162 (1982).

[43] K. Schreckenbach, G. Colvin, W. Gelletly and F. Von Feilitzsch, "Determination Of The Anti-neutrino Spectrum From ${ }^{235}$ U Thermal Neutron Fission Products Up To 9.5 Mev,” Phys. Lett. B 160, 325 (1985).

[44] A. A. Hahn, K. Schreckenbach, G. Colvin, B. Krusche, W. Gelletly and F. Von Feilitzsch, "Anti-neutrino Spectra From ${ }^{241} \mathrm{Pu}$ and ${ }^{239} \mathrm{Pu}$ Thermal Neutron Fission Products," Phys. Lett. B 218, 365 (1989).

[45] N. Haag, F. von Feilitzsch, L. Oberauer, W. Potzel, K. Schreckenbach and A. A. Sonzogni, "Re-publication of the data from the BILL magnetic spectrometer: The cumulative $\beta$ spectra of the fission products of ${ }^{235} \mathrm{U},{ }^{239} \mathrm{Pu}$, and ${ }^{241} \mathrm{Pu}$," arXiv:1405.3501 [nucl-ex].

[46] N. Haag, A. Gütlein, M. Hofmann, L. Oberauer, W. Potzel, K. Schreckenbach and F. M. Wagner, "Experimental Determination of the Antineutrino Spectrum of the Fission Products of ${ }^{238} \mathrm{U}$," Phys. Rev. Lett. 112, no. 12, 122501 (2014)

[47] G. Mention, M. Fechner, T. Lasserre, T. A. Mueller, D. Lhuillier, M. Cribier and A. Letourneau, "The Reactor Antineutrino Anomaly," Phys. Rev. D 83, 073006 (2011)

[48] A. de Gouvea and T. Wytock, "Light Sterile Neutrino Effects at $\theta_{13}$-Driven Reactor Neutrino Experiments,” Phys. Rev. D 79, 073005 (2009)

[49] A. C. Hayes, J. L. Friar, G. T. Garvey, G. Jungman and G. Jonkmans, "Systematic Uncertainties in the Analysis of the Reactor Neutrino Anomaly,” Phys. Rev. Lett. 112, 202501 (2014)

[50] G. T. Garvey, A. C. Hayes, G. Jungman and G. Jonkmans, "Further investigation of the "reactor anomaly," AIP Conf. Proc. 1666, 080001 (2015). 
[51] P. Vogel, G. K. Schenter, F. M. Mann and R. E. Schenter, "Reactor Anti-neutrino Spectra and Their Application to Anti-neutrino Induced Reactions. 2.," Phys. Rev. C 24, 1543 (1981).

[52] P. Huber, "On the determination of anti-neutrino spectra from nuclear reactors," Phys. Rev. C 84, 024617 (2011) Erratum: [Phys. Rev. C 85, 029901 (2012)]

[53] T. A. Mueller et al., "Improved Predictions of Reactor Antineutrino Spectra," Phys. Rev. C 83, 054615 (2011)

[54] J. Kopp, P. A. N. Machado, M. Maltoni and T. Schwetz, "Sterile Neutrino Oscillations: The Global Picture," JHEP 1305, 050 (2013)

[55] C. Giunti, M. Laveder, Y. F. Li and H. W. Long, "Pragmatic View of Short-Baseline Neutrino Oscillations,” Phys. Rev. D 88, 073008 (2013)

[56] C. Giunti and M. Laveder, "Statistical Significance of the Gallium Anomaly," Phys. Rev. C 83, 065504 (2011)

[57] J. Ashenfelter et al. [PROSPECT Collaboration], "The PROSPECT Physics Program," arXiv:1512.02202 [physics.ins-det].

[58] K. M. Heeger, B. R. Littlejohn, H. P. Mumm and M. N. Tobin, "Experimental Parameters for a Reactor Antineutrino Experiment at Very Short Baselines,” Phys. Rev. D 87, 073008 (2013)

[59] J. Ashenfelter et al. [PROSPECT Collaboration], "PROSPECT - A Precision Reactor Oscillation and Spectrum Experiment at Short Baselines,” arXiv:1309.7647 [physics.ins-det].

[60] N. Ryder [SoLid Collaboration], "First results of the deployment of a SoLid detector module at the SCK-CEN BR2 reactor," PoS EPS -HEP2015, 071 (2015)

[61] V. Hélaine, "Sterile neutrino search at the ILL nuclear reactor: the STEREO experiment," arXiv:1604.08877 [physics.ins-det].

[62] J. Ashenfelter et al. [PROSPECT Collaboration], "Background Radiation Measurements at High Power Research Reactors,” Nucl. Instrum. Meth. A 806, 401 (2016)

[63] I. Alekseev et al., "DANSS: Detector of the reactor AntiNeutrino based on Solid Scintillator," arXiv:1606.02896 [physics.ins-det].

[64] Y. F. Li, J. Cao, Y. Wang and L. Zhan, "Unambiguous Determination of the Neutrino Mass Hierarchy Using Reactor Neutrinos,” Phys. Rev. D 88, 013008 (2013)

[65] F. An et al. [JUNO Collaboration], “Neutrino Physics with JUNO,” J. Phys. G 43, no. 3, 030401 (2016) 